\title{
A procedure for collecting electromagnetic data using multiple transmitters and receivers capable of deep and focused exploration
}

\author{
Joshua Lymburner ${ }^{1}$ and Richard S. Smith ${ }^{2}$
}

\begin{abstract}
Many ground controlled-source electromagnetic (EM) systems have been deployed, and under ideal conditions these systems are capable of detecting large conductors to depths of approximately $800 \mathrm{~m}$; however, more common detection limits are less than $400 \mathrm{~m}$. Although these systems have been used with great success, they may experience two weaknesses when exploring for deeper conductors: poor coupling with the target and small signal-tonoise ratios (S/Ns), both of which decrease the quality and interpretability of the data. We evaluated a novel time-domain EM procedure that addresses these weaknesses. The coupling weakness was addressed through multiple transmitter locations and multiple receiver locations, and the $\mathrm{S} / \mathrm{N}$ was increased by spatial stacking of measurements (from the various transmitter-receiver combinations). A field test of this procedure was undertaken. Reciprocity
\end{abstract}

\begin{abstract}
data indicated that the noise levels of the vertical component data we acquired were about $-0.004 \mu \mathrm{V} / \mathrm{Am}^{2}$. Spatial stacking of the data can reduce the noise levels by a factor of seven. This means that a small conductor previously only visible to $150 \mathrm{~m}$ could be seen to $275 \mathrm{~m}$ and a conductor visible to $300 \mathrm{~m}$ could be seen to $575 \mathrm{~m}$. One challenge of the new procedure was the time required to collect all the transmitter-receiver combinations - this time can be reduced using the principle of reciprocity and not repeating approximately reciprocal measurements. Another challenge was to visualize and interpret the large volumes of data collected using the procedure - this has been partially addressed by creating equivalent-dipole depth sections. Synthetic and real equivalentdipole depth sections appeared very similar and illustrated that these images of the subsurface could be interpreted. However, the features appeared too deep on the sections, so better visualization techniques could be developed.
\end{abstract}

\section{INTRODUCTION}

As shallow mineral deposits become scarcer, the development of new techniques and methods is becoming essential to discover deposits at greater depths. In ideal circumstances, controlled-source ground-based electromagnetic (EM) systems can see to approximately 800-m depth (Koch and Dalidowicz, 1996; Nimeck and Koch, 2008), although most conductive targets are a challenge to identify when they are greater than a few hundred meters deep. Within the Sudbury Basin, EM methods have played a significant role in discovering nickel (Ni), copper $(\mathrm{Cu})$, and platinum group element (PGE) deposits in the Sudbury Igneous Complex (SIC) as well as within its footwall and within associated dikes (e.g., Polzer, 2000). Discovery of deeper deposits requires a transmitter configuration that will excite currents within these deeper targets. Often, this is accomplished using a larger transmitter loop, as the magnetic field will be stronger at depth and hence will provide an increased depth of penetration (Spies, 1989). However, to double the depth of exploration without increasing the loop size, an increase in the strength of the transmitter current by one order of magnitude is needed, in turn requiring the transmitter power to be increased by two orders of magnitude (Zhdanov, 2010). Indeed, high-powered transmitters are often deployed where access allows. However, issues with primary-field coupling to the target still occur. For example, to couple to a vertical body the transmitter must be offset a relatively large distance from the body, and this reduces the strength of the field at the target body location. Without definite knowledge of the depth, geometry, and orientation of the conductor, a single large loop may not always provide

\footnotetext{
Manuscript received by the Editor 27 May 2014; revised manuscript received 13 August 2014; published online 26 November 2014

${ }^{1}$ Formerly Laurentian University, Department of Earth Sciences, Sudbury, Ontario, Canada; presently Crone Geophysics and Exploration, Mississauga, Ontario, Canada. E-mail: jlymburner@hotmail.com.

${ }^{2}$ Laurentian University, Department of Earth Science, Sudbury, Ontario, Canada. E-mail: rssmith@laurentian.ca.

(C) 2014 Society of Exploration Geophysicists. All rights reserved.
} 
good coupling. The coupling issue has been partially addressed by the InfiniTEM $®$ system configuration (Malo Lalande, 2007), which uses a dual-loop configuration similar to that suggested by Spies (1975). This system is primarily designed for strong coupling to vertical plate-like bodies. However, if this loop is not properly placed, issues with poor coupling may still occur. As an alternative to the InfiniTEM configuration, Nimeck and Koch (2008) suggest laying out the two loops and measuring data from each loop separately, then combining the data (spatial stacking) after the survey to increase the signal-to-noise ratio $(\mathrm{S} / \mathrm{N})$. However, they acknowledge that this increases the measurement time by a factor of two. Powell et al. (2007) suggest using a stepwise moving-loop configuration, which has threefold coverage or three transmitters per receiver station. This configuration is not commonly used because of the difficulty of moving three large loops through the bush, but Powell et al. (2007) argue it is necessary to explore to great depths in the Athabasca Basin.

Another common method to see deeper targets is through longer acquisition times. Currently available EM surveys often use a single receiver that is moved among stations. The receiver only takes measurements at the station for a short period of time before being moved to the next location. Data collection over longer intervals allows the reduction of noise through repetitive temporal stacking. Through longer stacking times, the increase in the $\mathrm{S} / \mathrm{N}$ is increased as the square root of the number of transmissions (for an assumed Gaussian noise), with the amount of energy expended proportional to the number of transmissions (Zhdanov, 2010).

The methods to increase the depth of exploration that are described above have practical limits. To produce a larger source field, more power is required, and this is usually achieved using larger, more powerful, transmitters. Power is often supplied via a generator, but the use of larger more powerful equipment is less practical in remote locations. Longer stacking times require a receiver to occupy a station for a longer interval of time, extending the length of the survey. As a result, the maximum power source and the length of the receiver stacking time are factors that should be considered when designing an EM survey. To significantly increase detection limits effectively requires either a modification of current methods or the use of a new methodology.

The goal of all time-domain electromagnetic (TDEM) surveys is to detect specific changes in conductivity of the subsurface produced by either geology or specific geologic processes. The goal in mineral exploration is usually to detect metallic ores that can have variable geometric parameters and depths. To obtain the best possible data, a transmitter and receiver layout is required to couple best with the body of interest and to increase its response. Although TDEM is used from a variety of platforms and with several configurations, in surface-based exploration some of the most common configurations are the in-loop, fixed-loop, and Slingram configurations (Frischknecht et al., 1991; Sheriff, 2002). The in-loop method involves laying out a large transmitter loop and taking a receiver measurement within the loop. For the next measurement, the transmitter and the receiver are both moved. The fixed-loop configuration has the transmitter in a single location and a roving receiver in multiple positions, generally along a grid, with locations inside or outside the loop, or both. This is the most commonly used method for mineral exploration in forested terrain, with loops ranging from $\sim 200 \mathrm{~m}$ up to $2 \mathrm{~km}$. The larger loops are used to increase the $\mathrm{S} / \mathrm{N}$ and to see deeper bodies. For the Slingram configuration, the transmitter and the receiver are moved at a fixed separation interval along the profile line. The fixed separation interval used during the survey is selected according to the required depth of exploration, with the separation being roughly one and a half to two times the desired depth of exploration (Eaton and Hohman, 1987). The in-loop and Slingram configurations are ideal for reconnaissance, as multiple transmitter locations ensure coupling to conductors of unknown position and orientation. However, this method often uses smaller loops, which allows for efficient movement of the transmitter, but smaller transmitter loops are unable to produce a large enough field for the depth of exploration required for many of the targets in mineral exploration. In contrast, the loops in fixed-loop surveys are capable of reaching the required depth of exploration; but they are often too large to be moved more than a few times. A more detailed discussion of EM configurations is presented by Nabighian and Macnae (1991).

Geophysical companies have recently started to implement and develop a variety to distributed array systems for electrical surveys. The distributed systems are capable of "exciting" the subsurface from a large variety of current injection points and collecting large amounts of data efficiently, a concept that had been implemented within the seismic industry for decades (Nimeck and Koch, 2008). Three distributed array systems (MIMDAS, TITAN-24, and Geoferret) evolved from the Anaconda system from the 1970s (Halverson et al., 1989). The MIMDAS system, developed by Mount Isa Mines Exploration in 1997 (Garner and Webb, 2000), is a 100channel distributed array system capable of collecting resistivity/ induced polarization (IP) and magnetotelluric (MT) data (Kingman et al., 2007). MIMDAS was followed by the TITAN-24 system, which is a resistivity/IP/MT system able to measure full waveform data (McMonnies and Gerrie, 2007). Both systems deploy an array of wired inline and crossline dipoles, which are connected to data acquisition units, then to a CPU via a local network (Boivin, 2007). Resistivity/IP data would be collected during the day using multiple current injection points, which can be configured in poledipole, dipole-dipole, or hybrid arrays (Goldie, 2007), whereas MT is collected at night using the inline and crossline dipoles to measure the electric field in the $E_{x}$ and $E_{y}$ directions (Garner and Webb, 2000). A single pair of $H_{x}$ and $H_{y}$ magnetometers is buried on site because it is assumed that the magnetic field will remain fairly constant over the survey area, and a remote reference magnetic station is often set up for noise rejection. Compared with the standard six to eight dipole systems, the detection limits of distributed array systems are much deeper ( $750 \mathrm{~m}$ using the resistivity/IP system), as larger source-receiver offsets are possible resulting in a larger " $N$ level," with good spatial resolution from the high density of measurements (e.g., $N=20$ compared with $N=6$ or 8 ) (Eaton et al., 2010).

Another recently developed distributed acquisition system for IP is the Newmont distributed IP data acquisition system (NEWDAS) system developed by Newmont. NEWDAS has characteristics from the TITAN-24 and the Geoferret system (described below). The transmitter source can be deployed as a pole-dipole, dipole-dipole, or hybrid array with wireless receiver capabilities. The multiple excitation sites and configurations allow for a greater depth of investigation through larger source-receiver offsets and high sample density. The advantage of wireless receivers is that any specific exploration goal can be addressed because the receivers are not bound to a fixed cable and receiver separation. The GPS-enabled receivers allow for synchronizing the transmitter and the receivers, and 
the wireless transmission of data makes deployment of the system easier and less cumbersome, although wired communication is an option (Eaton et al., 2010).

The Geoferret system is a distributed-receiver-array EM system developed by WMC Resources between 1999 and 2005 (Golden et al., 2006). This system was developed to rapidly acquire timedomain EM data at high spatial resolution and was designed to be capable of deep target detection. The system uses a wireless $z$ component receiver coil that was designed to have low noise. The survey design (Golden et al., 2006) used a large fixed-loop transmitter, whereas multiple receivers were deployed along the grid in an "array." The receivers occupy each station for about an hour while the transmitter runs continuously. This results in a measurement period that is approximately 10 times longer than conventional TDEM surveys (Kingman et al., 2007). The longer stacking times, coupled with the low-noise receiver, produced significant noise rejection, increasing the data quality and depth of exploration of the system (Golden et al., 2006). Multiple transmitter positions can be used in this design to provide additional coupling positions, although this process would be time consuming in comparison with resistivity/IP distributed array systems. Distributed array systems are not yet commercially available for ground EM systems, due to the high cost of developing a system with high sampling rates (e.g., 50-kHz sampling) and an ability to stack and/or store this high-bandwidth data.

When these distributed array EM systems eventually become available, we are proposing a survey methodology that combines data from multiple transmitter and receiver locations. Similar ideas have been proposed in the past (Polzer et al., 1989), but they did not gain traction, possibly due to using only three transmitter loops. By combining data from multiple transmitters, we can minimize the issues associated with poor coupling. The difficulty of moving large loops in the bush is addressed by using small loops with multiple turns. Data from multiple receivers can be combined to further enhance the S/N. This is loosely related to "beam forming" of seismic arrays or arrays of radio telescopes (Rost and Thomas, 2002), although we are not dealing with wave propagation and so phase delays are not used to guide the signals in certain directions in this experiment.

To produce a methodology that is effective in acquisition and detection, it must be able to effectively excite currents within deposits at depth, while quickly acquiring the best data possible. According to the reciprocity theorem (Harrington, 1961; Parasnis, 1988; Chen et al., 2005), the response should remain unchanged when a transmitter is replaced with a receiver that has a coil in exactly the same location as the transmitter coil and the receiver is replaced with a transmitter coil in the identical location as the receiver coil. Application of this theorem to time-domain, ground EM techniques means that not all transmitter-receiver combinations need to be collected if a reciprocal (and hence redundant) measurement has already been collected. This means that survey acquisition time can be reduced.

Using a methodology that deals with multiple transmitter and receiver locations will produce a larger amount of data that are difficult to visualize and interpret. Equivalent-dipole depth sections are one way of handling large data sets so that the data can be visually displayed as a function of position and depth and interpreted easily.

The S/N and hence the depth of exploration can also be increased by modifying the transmitter waveform shape and focusing the energy in a frequency range to which the target is sensitive (e.g., Commer et al., 2006). However, in this paper, we are focusing on obtaining improvements through modification of the number of transmitters and receivers; if changes in waveform shape provide further improvements, then we assume these can be applied in addition to our proposed changes.

\section{DATA ACQUISITION}

A test site was selected with a target at approximately 70-m depth in the northeast range of the Sudbury Basin, approximately $3 \mathrm{~km}$ northeast of the SIC within the rocks of the Superior Province. The target is enclosed in the north-south-trending mafic and felsic Archean metavolcanic rocks with subvertical dips and several metagabbro dikes (Tirschmann, 1990). Felsic metavolcanic rocks are located to the west of the property, and the central region is underlain by massive feldspar porphyry and quartz-feldspar porphyritic rocks.

The Parkin quartz diorite dike is continuous across the property trending at an azimuth of $33^{\circ}$ with its width ranging from 45 to $135 \mathrm{~m}$ and is believed to be a faulted extension of the Whistle offset dike (Croteau, 2009). The small, vertical, thin deposit chosen to test this method is consistent with other dike deposits and consists of massive to patchy pyrrhotite and pyrite with blebs, disseminated fracture fillings, and lenses of chalcopyrite in siliceous fragments as the dominant portion of disseminated Cu-PGE mineralization (Croteau, 2009). This is not a deep target, but it was an easily accessible target that could be used for experimenting with procedures that could be used to look for deeper targets.

The time-domain survey described in this paper was carried out over a $1-\mathrm{km}$ line at a station spacing of $25 \mathrm{~m}$, with the known deposit located approximately in the middle of the line. The survey was completed using a TEM57-MK2 transmitter, two 10 by $10 \mathrm{~m}$ loops (each with 10 turns), and two receiver teams each using a SMAR$\mathrm{TemV}$ receiver with a Geonics $3 \mathrm{D}$ induction coil sensor. The current waveform was a square wave with a peak current amplitude of $20 \mathrm{~A}$, and the base frequency was $30 \mathrm{~Hz}$.

To test the concept of using multiple transmitters to improve coupling, the transmitter was placed at all stations in the inner $600 \mathrm{~m}$ of the line, accounting for 24 transmitter positions. Figure 1 is a simplified schematic of the survey line. Because the location of the mineralized zone was known, the positions were chosen to simulate locations that would both couple well and poorly to and from the
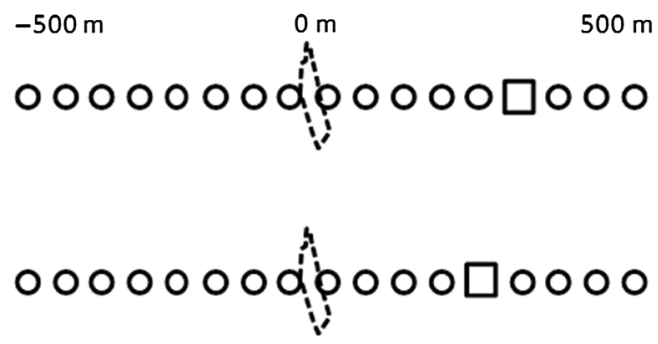

Figure 1. Simplified schematic plan view of survey logistics. Rectangles represent the transmitter position, circles represent the receiver positions, and the dashed line represents the outline of the ore body at approximately $75-\mathrm{m}$ depth. In reality, there were 51 receiver positions and a total of 25 transmitter positions. Receiver measurements were recorded every $25 \mathrm{~m}$, whereas the 24 transmitter positions only occupied the inner $600 \mathrm{~m}$ of the survey line. 
body. We do not have a distributed array acquisition system to test the concept of using multiple receivers, so long stacking times could not be used in our experiment. However, we did have access to two receivers, so these were both deployed to reduce the acquisition time by half. We used industry standard stack times of a few minutes. At each transmitter position, the two receiver teams would each survey half of the survey line, so that together the whole line would be surveyed. The transmitter was then moved to the next location, and the receiver teams each repeated the measurements on half of the survey line. This procedure was repeated until all 24 transmitter locations had been occupied. We had enough wire for two transmitter loops, which allowed a slight decrease in the setup time because the unused loop could be positioned at the next station while the other loop was active as the transmitter. Binding and color

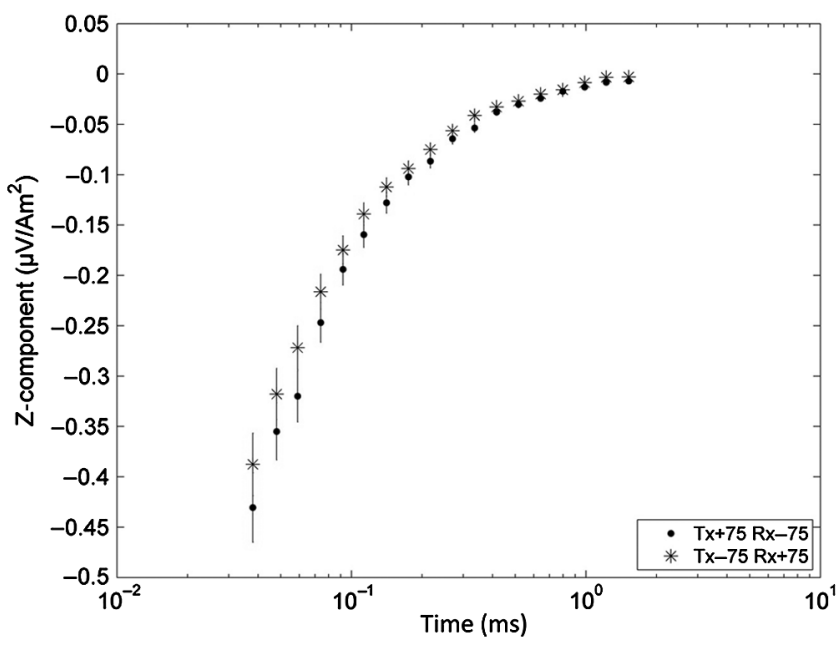

Figure 2. Measurements for two reciprocal transmitter-receiver locations $(T x+75 \mathrm{~m}, R x-75 \mathrm{~m}$ and $T x-75 \mathrm{~m}, R x+75 \mathrm{~m})$. Both sets of measurements agree to within the combined estimated noise of $8 \%$ represented by the vertical error bars. The results in windows 3-20 are shown.

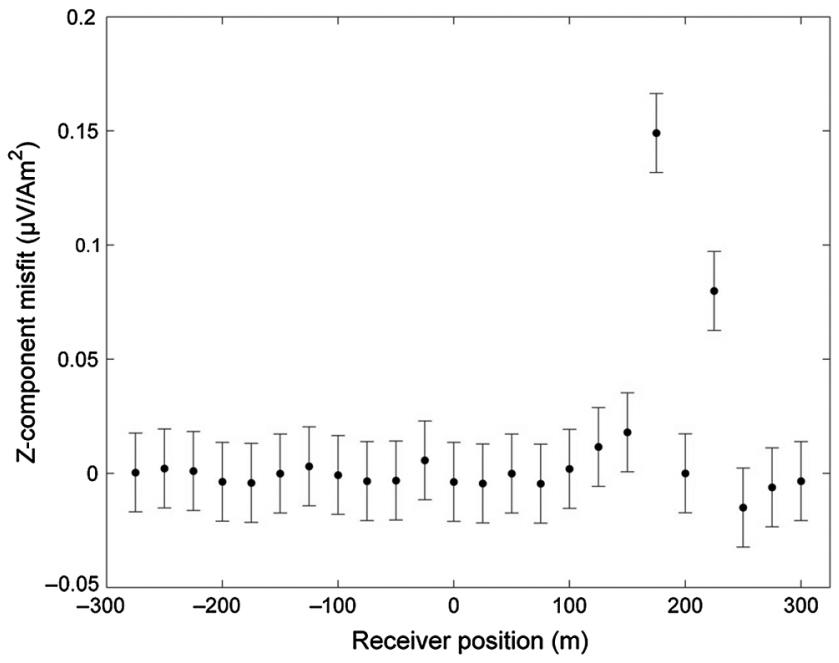

Figure 3. The misfit in the $z$-component (average of windows $3-20$ ), of all the reciprocal measurements for transmitter position $+200 \mathrm{~m}$. coding the 10 parallel wires together meant that all 10 turns could be laid out at once. This simplified loop deployment in areas with thick vegetation and rugged terrain.

\section{RECIPROCITY MEASUREMENTS}

Reciprocity tests were conducted through the analysis of transmitter and receiver pairs that are approximately reciprocal. Each of these pair was assumed to give the same measurement, and the difference was analyzed to determine the amount of error associated with the measurement. The reciprocity theorem (Parasnis, 1988) says that if you leave the transmitter and receiver coils in place and make the transmitter coil a receiver coil (and vice versa), then the measurement should be identical. In this case, the transmitter is approximately $10 \mathrm{~m}^{2}$ and the receiver $1 \mathrm{~m}^{2}$. However, the change in area has been normalized for and the different shape is not significant when the transmitter and the receiver are more than 30-m apart.

Independent estimates of the expected noise were also obtained by analyzing other sources of noise, including geometric noise associated with the transmitter (orientation and geometry). Conservative estimates of a $\pm 15 \mathrm{~cm}$ change in the geometry of the transmitter loop (from $10.15 \times 10.15 \mathrm{~m}$ to $9.85 \times 9.85 \mathrm{~m}$ ) and a $5 \%$ change in the slope of the topography yielded a net noise estimate of approximately $8 \%$. Figure 2 shows an example of a reciprocal pair of $z$-component measurements that fall within the combined noise estimate $(8 \%)$, represented by the vertical error bars. In this case, the average misfit of the response at all the delay times for this pair is $0.015 \mu \mathrm{V} / \mathrm{Am}^{2}$. In this data set, the early time windows (1-3) appear to be corrupted, possibly by poor sampling or by transmitter ramp effects, so windows one and two were not shown in this plot, and the first three windows have been removed from the analysis below.

There is a multiplicity of transmitter-receiver reciprocal pairs, and the misfit has been calculated for all of these. Displaying all these results on one plot is difficult: As an example of some of the misfits, Figure 3 shows the misfits associated with all the pairs that are associated with transmitter position $+200 \mathrm{~m}$.

The misfit among most of these reciprocal pairs falls well within a noise level of $0.02 \mu \mathrm{V} / \mathrm{Am}^{2}$. However, two data points are above the calculated noise. This trend is evident in all of the other transmitter plots, with large misfits being observed on either side of the transmitter position. The close proximity of the transmitter is believed to result in the voltage being so large that it has saturated the analogue to digital converter, causing the measurements to become corrupted. Another possibility is that the transmitter and the receiver are close so that the large and small loops cannot be considered to be the same shape, so that the pair is not a true reciprocal pair.

A summary plot (Figure 4) was created from the average misfit of all the transmitter positions to determine the total noise associated with the receiver electronics, the transmitter, and the relative geometry. Most of the data exhibit a $z$-component misfit of around $\pm 0.004 \mu \mathrm{V} / \mathrm{Am}^{2}$, and all of the misfits are between $\pm 0.01 \mu \mathrm{V} / \mathrm{Am}^{2}$. In this figure, corrupted windows and measurements in which the receiver is too close to the transmitter have been removed. Because the reciprocal measurements agree to values that are within the expected misfit, reciprocity has been demonstrated for the pairs that have not been removed.

Although many reciprocal measurements were conducted during this experiment, if reciprocal measurements were not acquired, it 
would have reduced the acquisition time substantially. In total, 960 measurements were acquired over the $1-\mathrm{km}$ line with $25-\mathrm{m}$ station receiver spacing and 24 different transmitter locations (measurements were not taken within the transmitter due to the data being corrupted). Of these, 276 measurements were approximate reciprocals, so not acquiring them would result in a $29 \%$ reduction in the number of stations occupied. Because the transmitter is moved from one location to the other down the line, the previous spot occupied by the transmitter becomes a reciprocal measurement for the next transmitter position. Figure 5 shows the station locations that should be collected and those that can be omitted through reciprocity. The vertical axis is the receiver position, the horizontal axis is the transmitter position, and each column is the stations that need to be occupied (circles) or do not need to be occupied (blank) on the survey line. The first transmitter position occupied on the survey was at location $+300 \mathrm{~m}$ (see the column on the extreme right of the plot). For this transmitter, all receiver locations had to be occupied. For the next transmitter at $+275 \mathrm{~m}$ (second column from the right), it was not necessary to place the receiver at station $+300 \mathrm{~m}$, as there was already a reciprocal measurement with the receiver at $+275 \mathrm{~m}$ and the transmitter at $+300 \mathrm{~m}$. As more transmitter positions are occupied farther down the line, fewer receiver positions must be occupied back up the line. Eventually, at the last transmitter position, all previous transmitter locations become reciprocal measurements. The measurements that still need to be acquired are those in locations that were not occupied by the transmitter (i.e., those in locations that are in the $200 \mathrm{~m}$ on the two extreme ends of the line). These stations account for a total of 456 stations over the course of the survey. With two receiver teams, each transmitter move will reduce the number of receiver stations that must be measured on the line by one station. Having each receiver team move up and down the line from the middle can reduce the number of measurements, especially toward the last transmitter positions, because only the ends of the line need to be surveyed amounting to 17 measurements. If reciprocity was invoked during the survey described in this paper, it may have reduced the acquisition time by a day or two. If reciprocity is not invoked for the case when the transmitter is close to the receiver, then the time savings would not be quite as great.

If there had been multiple receivers (e.g., an array of 51 receivers with one receiver at each station on the line) then the survey time would have been significantly less.

\section{COMBINING MEASUREMENTS}

We investigate the consequences of combining the measurements at a particular receiver location from one transmitter with the measurements at the same receiver location and one or more other transmitters. Doing so can raise the signal of a subsurface conductor above the noise threshold and allow it to be detected. This will be demonstrated using different transmitter combinations that simulated larger loops as well as other configurations such as the InfiniTEM configuration. Our transmitter locations were only along one traverse; if there were transmitter positions off-line, there will be a larger number of possible transmitter combinations and a greater increase in the signal.

Using multiple transmitter locations ensures that some of the transmitters will couple well with the body regardless of the location of the body in the subsurface. For example, in Figure 6, absolute coupling coefficients (normalized by the peak amplitude) were calculated as a function of transmitter offset for a 50-m deep vertical conductor (horizontal dipole) in free space located at three locations on the profile, 100,0 , and $-100 \mathrm{~m}$. In all three scenarios, the body and the transmitter are null coupled when the transmitter is directly over the body. However, as the transmitter offset is increased, the coupling increases to a maximum about $25 \mathrm{~m}$ away, followed by a decrease in coupling with larger offsets. In this example, placing the transmitters every $100 \mathrm{~m}$ (e.g., at $-300 \mathrm{~m},-200 \mathrm{~m}, \ldots 300 \mathrm{~m}$ ) would only give coupling coefficients of about 0.1 or less, a poor result. However, choosing transmitter positions every $50 \mathrm{~m}$ (e.g., at $-300 \mathrm{~m},-250 \mathrm{~m}, \ldots 300 \mathrm{~m}$ ) would ensure coupling coefficients of greater than 0.6 at more than one location for each conductor. This is a much better result.

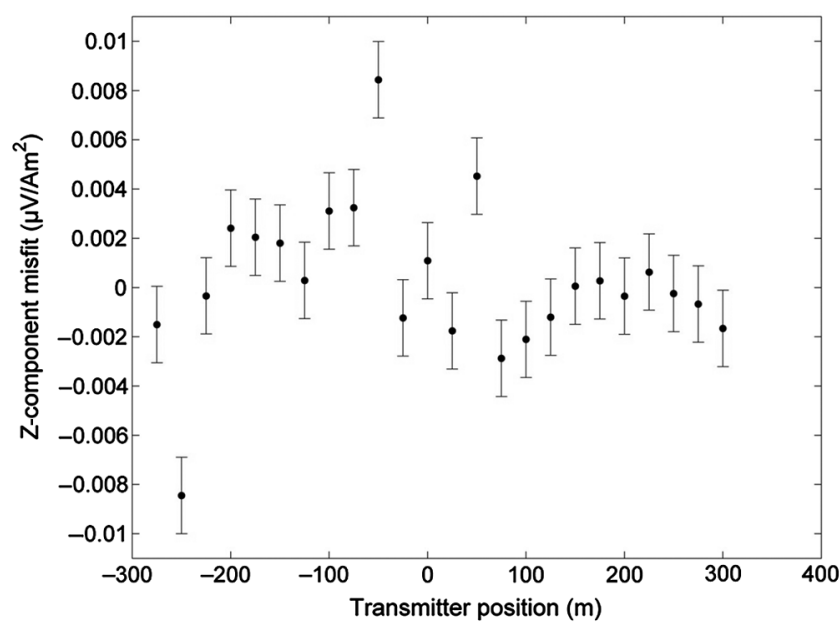

Figure 4. Summary plot of the average misfit for reciprocal pairs, as a function of the transmitter position. Corrupted data have been removed to provide a better estimate of the noise.

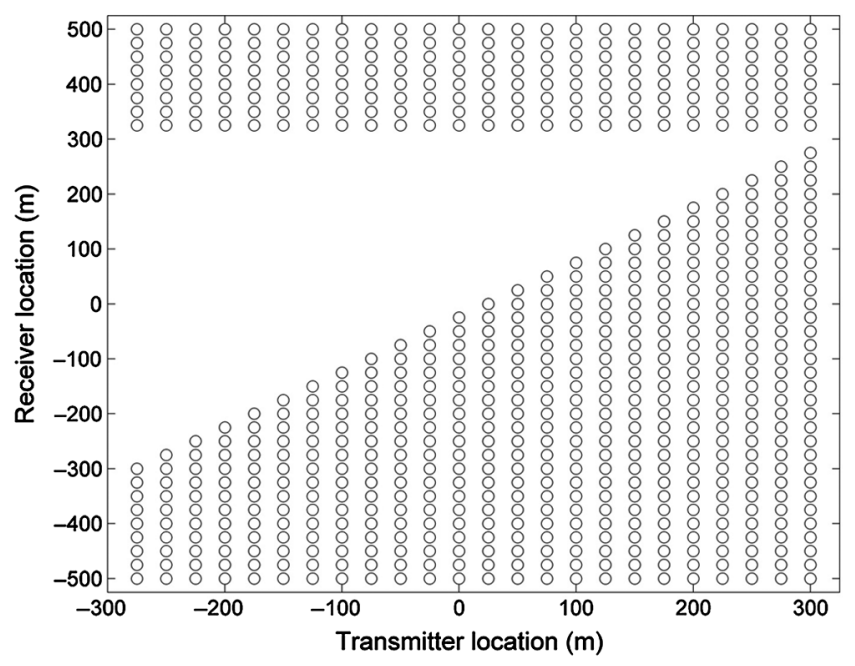

Figure 5. The location of receiver positions that would need to be measured as a function of transmitter position if reciprocity was applied. The circles represent the location of the measurements that would need to be taken to produce the same data gathered during the survey described in this paper. The white area represents the measurements that can be omitted if reciprocity was invoked for the survey. 


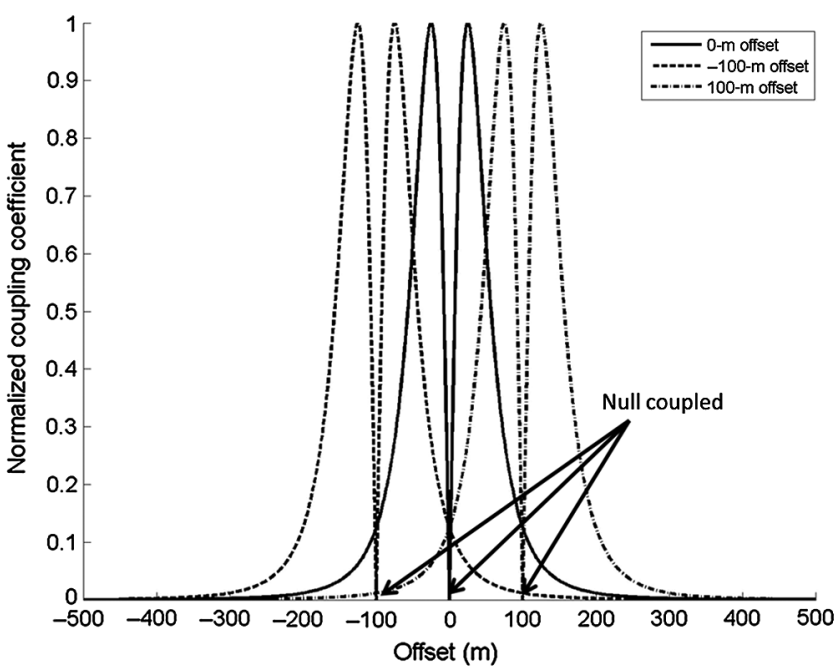

Figure 6. Absolute value of the calculated normalized coupling coefficients ( $z$-component) as a function of transmitter offset with respect to a horizontal dipole at a depth of $50 \mathrm{~m}$. The solid line represents a body at 0 - $\mathrm{m}$ offset, the dashed-dotted line is for a body at 100-m offset, and the dashed line is for a body at $-100-\mathrm{m}$ offset. Directly above the body, the transmitter is null coupled, but with the transmitter offset increasing maximum coupling is achieved at $25 \mathrm{~m}$ either side of the body followed by a decrease in coupling with larger offsets.

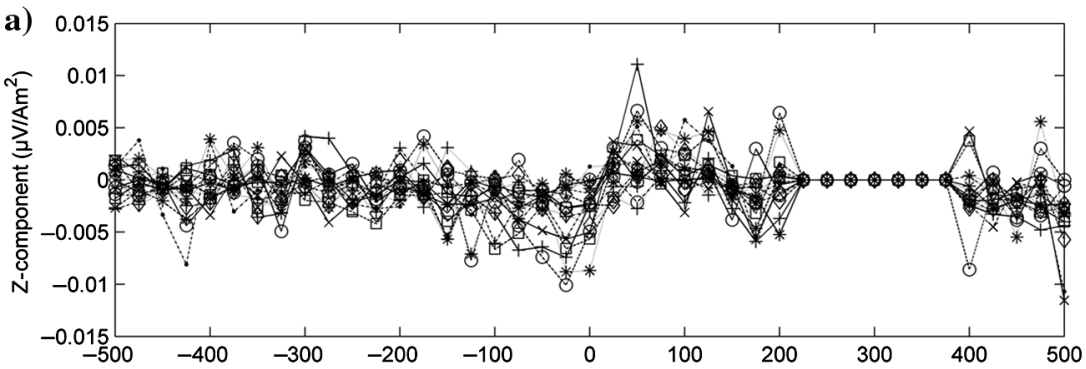

b)
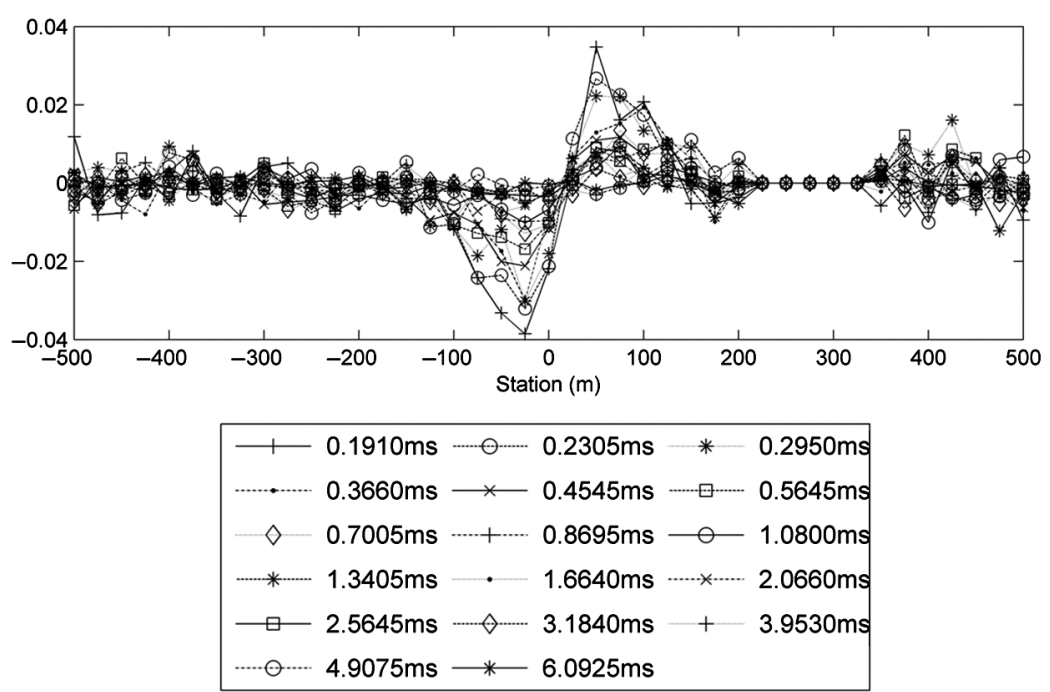

Figure 7. The amplitude of the response plotted as a function of receiver position. The (a) is for a transmitter at location $+300 \mathrm{~m}$, and (b) shows the summed response from three transmitter locations between +300 and $+250 \mathrm{~m}$. The delay times on the legend are in millisecond after the transmitter current has completely switched off.
The reciprocity data and an examination of the magnitude of the geometric errors suggest that the total noise for each transmitterreceiver pair is approximately $0.01 \mu \mathrm{V} / \mathrm{Am}^{2}$ or less (when corrupted receiver locations close to the transmitter are removed). The responses at one receiver location for one transmitter can be added to the response at the same receiver location for a second transmitter, to give the larger signal that would be obtained if a transmitter that is two times larger were used. If the noise is random in sign, the summed noise will decrease and hence the S/N will increase. Figure 7 shows a profile with one transmitter at location $+300 \mathrm{~m}$ and another profile when receiver measurements from three transmitters $($ at $+300,+275$, and $+250 \mathrm{~m})$ are added. This addition process is a simple summation. If all the receiver measurements are column elements within the row of a matrix, then one row is for the transmitter at $+300 \mathrm{~m}$, with other rows for other transmitter positions. The data in the bottom panel of Figure 7 were generated by summing the three rows associated with transmitter positions +300 , +275 , and $+250 \times \mathrm{m}$. When the transmitter is at $+300 \mathrm{~m}$ (top panel), the response of the body is just evident above the noise level on this line, which appears to be about $0.005 \mu \mathrm{V} / \mathrm{Am}^{2}$. The response falls below the noise by window six, so that the decay is interpretable up to this delay time $(0.2950 \mathrm{~ms}$ after switch off). The $\mathrm{S} / \mathrm{N}$ at $0.1910 \mathrm{~ms}$ (window four) is 1.11 . Windows $1-3$ were not plotted because the data may have been corrupted due to poor sampling of the early time decay or transmitter ramp effects. The area with no data displayed represents locations in which the receiver response appears to be corrupted due to the close proximity of the transmitter. The response obtained by summing the response from three transmitter positions from +300 to $+250 \mathrm{~m}$ inclusive (bottom panel) gives an $\mathrm{S} / \mathrm{N}$ of 3.86 at $0.1910 \mathrm{~ms}$, and the decay remains above the noise to window 10 , giving an interpretable decay until $0.7005 \mathrm{~ms}$.

The increase in the $\mathrm{S} / \mathrm{N}$ for this process is dependent on the number of transmitter positions summed and the coupling of each transmitter. With more transmitter positions summed together, the response is increased and the noise is reduced. Figure 8 shows how the sum of four adjacent transmitter locations $(+300$ to $+225 \mathrm{~m})$ that are well and poorly coupled can simulate a larger loop, increasing the response from the body. The response so obtained gives an $\mathrm{S} / \mathrm{N}$ of 6.98 at $0.1910 \mathrm{~ms}$, and the decay remains above the noise up to window 11 , giving an interpretable decay until $0.8695 \mathrm{~ms}$. Part of the reason for the increase in the $\mathrm{S} / \mathrm{N}$ is due to noise reduction due to spatial stacking, and part is due to the fact that the coupling is greater because the responses associated with the transmitters that have been added are large because the transmitter is closer and couples more strongly to the target.

The impact of the spatial stacking alone can be estimated by stacking the results from transmitters, which are equidistant from the target and have similar coupling. Figure 9 shows the profiles from the transmitters at the ends of the line (at +275 and $-275 \mathrm{~m}$ on the top and middle 
panels, respectively). The anomaly in each of these two panels is ambiguous: in the top panel the negative lobe to the left is larger than the right positive lobe, whereas in the middle panel the positive lobe to the right is larger. For fixed-loop data such as these, the response shape of a thin plate-like conductor will not change significantly with transmitter position. For the subvertical conductor that is below this profile line, we expect a symmetric response, like that seen in Figures 7, 8, and 10. The summed profile in the bottom panel of Figure 9 is more symmetric than the top or middle panels, so it provides a better profile to interpret. Note that in each of the three panels of Figure 9, the scales have been selected so the signal in each case is roughly the same size, so that the $\mathrm{S} / \mathrm{N}$ envelope can be perceived to be slightly less on the bottom panel. The S/N on the bottom panel is 2.6 at $0.1910 \mathrm{~ms}$, and the decay remains above the noise up to window eight, giving an interpretable decay until $0.4545 \mathrm{~ms}$. This illustrates that combining the data from many poorly coupled positions can still be of value because the stacking may elevate the response above the noise envelope.

The act of stacking together data from multiple transmitters is equivalent to making the transmitter loop larger. Thus, the resulting stacked data can be interpreted without error by modeling the response as from a larger (summed) transmitter loop.

If the dip of the body were different, different combinations would give a stronger S/N. For example, for a horizontal thin sheet, a large response could be obtained by summing the transmitters directly above the body. If the sheet is vertical, then transmitters on either side of the body will couple in opposite ways, so subtracting the receiver responses associated with transmitters on one side from the receiver responses from another set of transmitters on the opposite side will enhance the response. This is equivalent to having the current in the transmitter flowing in opposite directions around the loop, which is essentially what happens in the InfiniTEM configuration used by Abitibi Geophysics (Malo Lalande, 2007). Figure 10 shows how transmitters on either side of the body can be combined in an InfiniTEM configuration so as to significantly increase the response from a vertical conductor. In this example, the receiver response associated with transmitter positions +75 to $+150 \mathrm{~m}$ are summed and then subtracted from the sum associated with transmitter positions -75 to $-150 \mathrm{~m}$. This is equivalent to summing four rows and subtracting the resultant from the sum of another four rows. The resulting $\mathrm{S} / \mathrm{N}$ is 144 at $0.1910 \mathrm{~ms}$, and the decay remains above the noise up to window 20 , giving an interpretable decay until $6.0925 \mathrm{~ms}$.

With multiple transmitter locations, the body can be excited from many different locations, online and offline. Exciting the body from multiple orientations and analyzing the different responses can reveal geometric information about the body, such as strike and dip. Furthermore, these offline transmitter positions would provide additional excitation by simulating a larger transmitter loop extending either side of the survey line and when these data are added to the sum would further increase the $\mathrm{S} / \mathrm{N}$. In many of the examples demonstrated, the increase in the $\mathrm{S} / \mathrm{N}$ of the receiver measurements is dependent on the position of the transmitter with respect to the body. For example, Figure 8 (discussed above) showed that by increasing the transmitter area by a factor of four increases the $\mathrm{S} / \mathrm{N}$ to a factor of seven.

It is possible to demonstrate how this increase in the $\mathrm{S} / \mathrm{N}$ due to combining transmitters can result in an ability to see deeper. Let us assume that there is a vertical body (horizontal dipole) buried at a depth of $150 \mathrm{~m}$, the response of which is just visible in data that

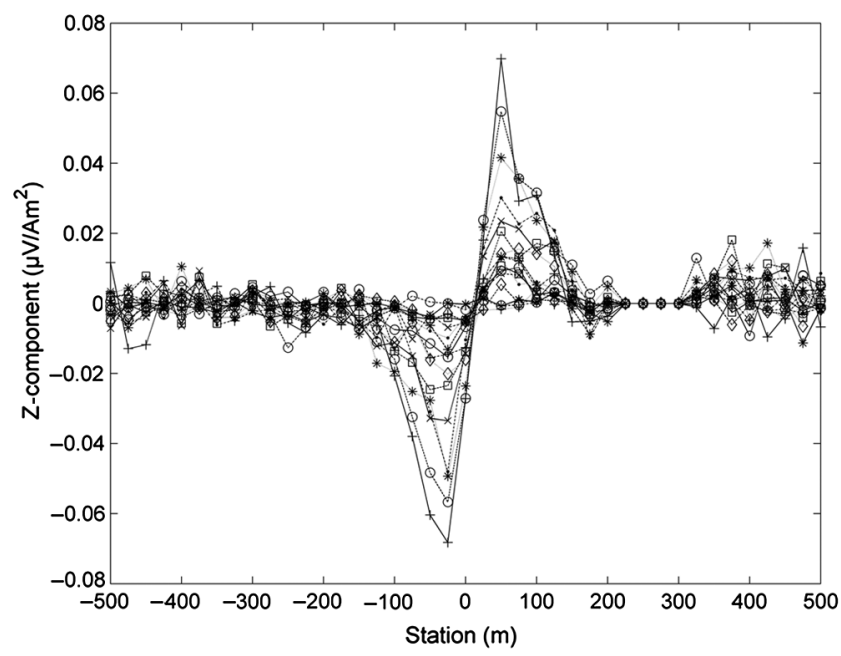

Figure 8. The amplitude response plotted as a function of receiver position for a summed response for transmitter locations between +300 and $+225 \mathrm{~m}$ inclusive. The legend is the same as in Figure 7.

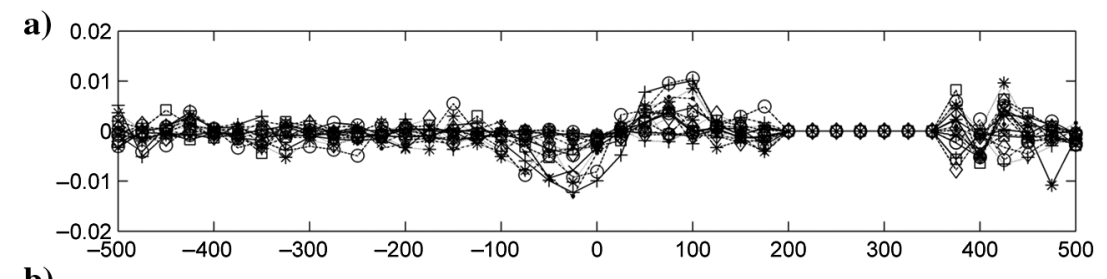

b)

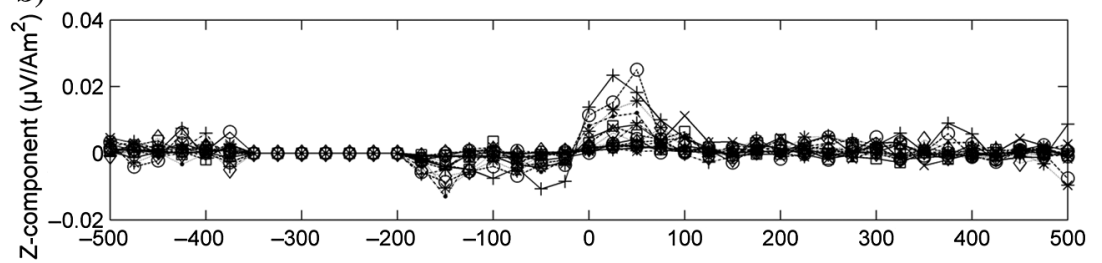

c)

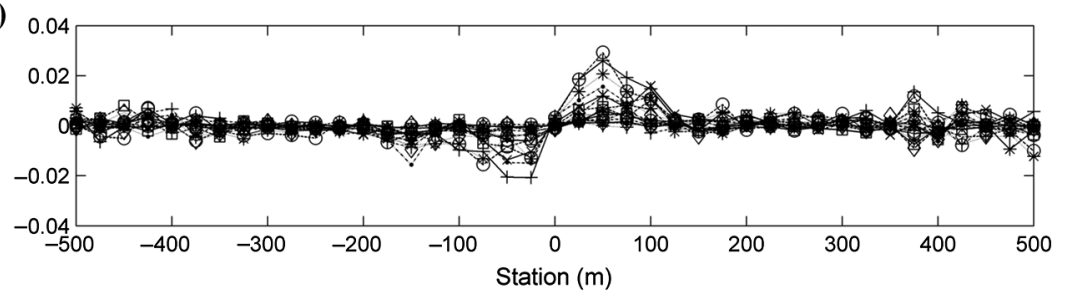

Figure 9. (a) The amplitude response plotted as a function of receiver position for transmitter location $+275 \mathrm{~m}$. (b) The amplitude response plotted as a function of receiver position for transmitter location $-275 \mathrm{~m}$. (c) The amplitude response plotted as a function of receiver position for transmitter locations $+275 \mathrm{~m}$ plus $-275 \mathrm{~m}$. The (c) is less affected by noise, and the size of the positive and negative lobes is more comparable. The legend is the same as in Figure 7. 
have not been combined. In this case, Figure 11 shows that the magnetic field of the dipole on the ground surface directly above the dipole will be about $3 \times 10^{-3} \mu \mathrm{V} / \mathrm{Am}^{2}$. If combining the transmitters results in a reduction in the noise level by a factor of seven, then the body should be visible when its magnetic field is reduced to $4.3 \times 10^{-4} \mu \mathrm{V} / \mathrm{Am}^{2}$. From Figure 11, it can be seen that the same dipole has this amplitude when it is at a depth of $300 \mathrm{~m}$. A similar argument shows that a stronger and deeper horizontal dipole at a depth of $300 \mathrm{~m}$ would be detected to $575 \mathrm{~m}$ when the transmitter measurements are combined. Hence, the act of combining transmitter measurements allows the $\mathrm{S} / \mathrm{N}$ to be increased and the depth to which bodies can be detected is increased.

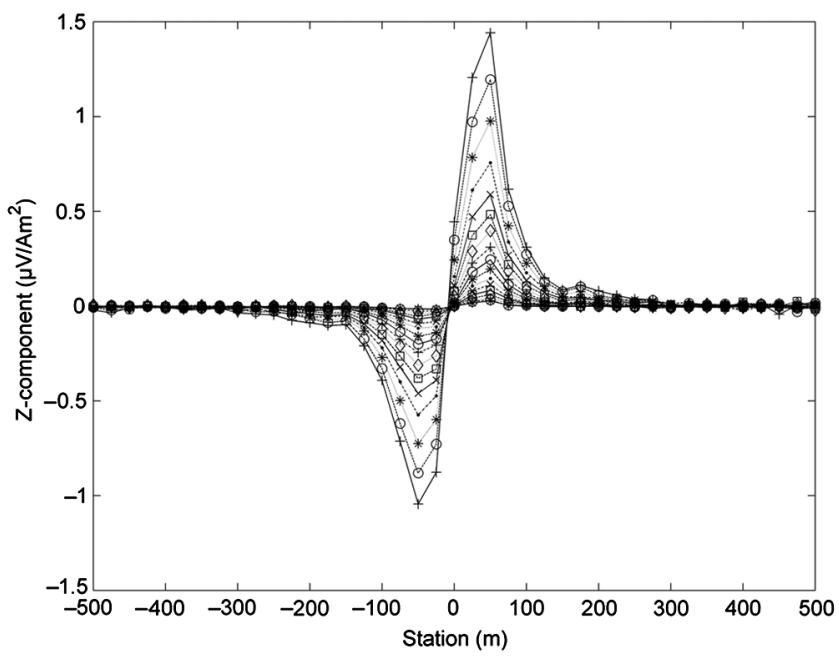

Figure 10. The amplitude response plotted as a function of receiver position obtained from summing the responses for transmitter locations +75 to $+150 \mathrm{~m}$ and subtracting the sum from locations -75 to $-150 \mathrm{~m}$. The legend is the same as in Figure 7 .

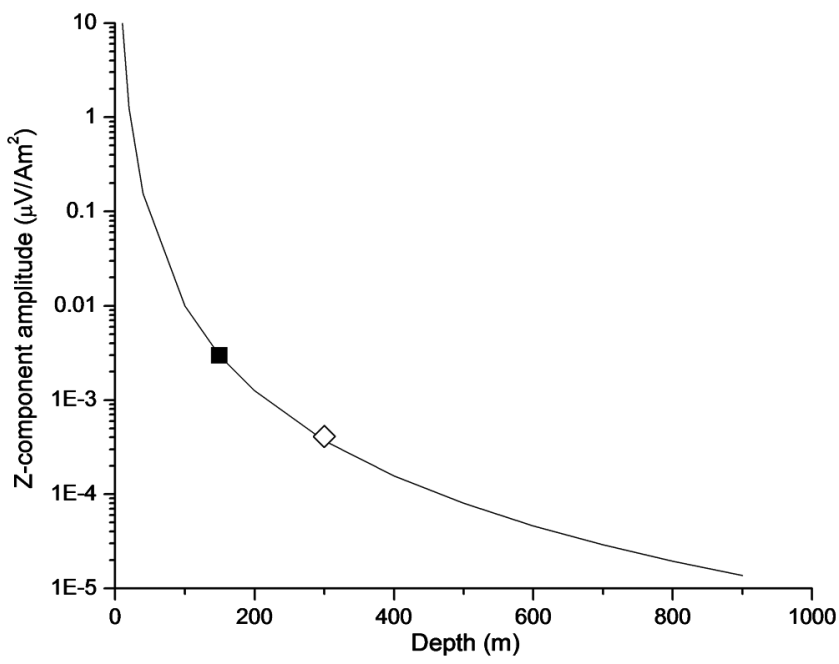

Figure 11. The decrease of the $z$-component response as a function of depth for a horizontal dipole. The square represents the amplitude response at a depth of $150 \mathrm{~m}$, whereas the diamond represents the depth the same body can be seen if the $\mathrm{S} / \mathrm{N}$ were increased by a factor of seven. The source moment of the horizontal dipole is $1 \mathrm{Am}^{2}$.

\section{EQUIVALENT-DIPOLE DEPTH SECTIONS}

The enormous volume of data and the variety of different transmitter-receiver combinations means that summarizing and interpreting all the data is a challenge. One way of summarizing the data and of creating an image that can be interpreted is to create an equivalent-dipole depth section. Another advantage of these sections is to generate multiple combinations of all the transmitters in such a way that the $\mathrm{S} / \mathrm{N}$ will be increased when the target body is at one of the multiple depths and locations. In this way, the section could show responses that might be coming from features at these multiple depths and locations.

In a previous section of the paper, the $\mathrm{S} / \mathrm{N}$ was increased by summing a few transmitters together. This is effectively a sum in which the transmitters included in the sum have an equal weight. For example, in the case in which three transmitters were summed, the weights were $+1,+1$, and +1 for the three transmitters and zero for the other transmitters. For the InfiniTEM example of Figure 10, the transmitter weights were $0,0,+1,+1,+1,+1,0,0,0,0,0,-1$, $-1,-1,-1,0$, and 0 (for transmitters -200 to $+200 \mathrm{~m}$ and zero elsewhere). These particular weights were selected to generate a strong response at a specific location and depth. Of course, there is an infinite variety of transmitter weights that could be applied. We propose to apply many different sets of weights that will constructively enhance the response from many different locations and depths. The specific form of the transmitter weights is selected to be proportional to the field from a horizontal dipole at a specific depth and location on the profile. This dipole field is then normalized by the peak field. For example, the shape of the weights will be similar to the curves on Figure 6 because they will provide a strong field at a depth of $50 \mathrm{~m}$ in three locations $(-100,0$, and +100$)$. However, they will not be positive on both sides of the null coupling position; like the InfiniTEM example, they will be positive on one side of the target and negative on the other. This transmitter weighting process is equivalent to multiplying each row of the matrix by a different set of weights for each location and depth combination and then summing the rows to give a resultant row for each location and depth.

Applying these transmitter weights will produce a response at each receiver that has a larger $\mathrm{S} / \mathrm{N}$ as it combines many transmitter positions. If there is a horizontal dipole at the specific depth and location that we are investigating, then it will have a response that looks like the field from the horizontal dipole. If we can estimate how much this response looks like the expected response, then we can come up with a measure of whether or not there is a dipole-like body at this depth and location. We propose that one measure of whether the response has the expected shape is to multiply receiver locations where we expect the response to be large by a large weight and where we expect the response to be small by a small weight; we then sum all the weighted receiver responses together to give a summed response. Receiver responses that are large at the locations where the weights are large will constructively add to give a large summed response; receiver responses that are large where they are not expected to be large will be weighted to give a smaller summed response as the weights at these receiver locations are smaller. As well, the weights can contain equal amounts of positive and negative values, so they will generate a sum with a small value. The weights we used are the fields at the receiver location from a horizontal dipole at the subsurface location of interest, normalized by the maximum field at all the receiver locations. This process is equivalent to multiplying elements in the resultant row by weights 
and then summing all the elements to give a single scalar value for that location and depth. This value is assigned to that location and depth on the equivalent-dipole depth section.

This whole process is then repeated when we wish to investigate another depth and location. If the summed responses at all the different depths and locations are displayed at their specific depths and locations, then the result is an equivalent-dipole depth section. Large responses can be displayed as red and small responses as blue. Locations that are large correspond to depths and locations in which there is a response that is similar to the response of the horizontal dipole. Hence, red locations will highlight targets at locations where they might not have been known or expected, highlighting the fact that the multi-transmitter-receiver procedure does not need to know a priori the location of the target.

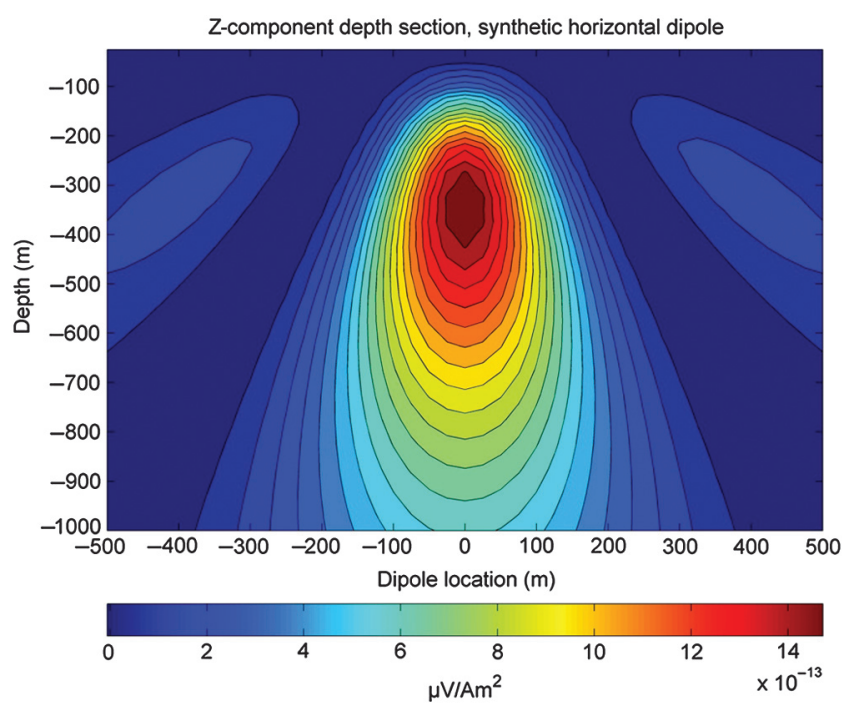

Figure 12. Synthetic equivalent-dipole depth section for a horizontal dipole located at $0 \mathrm{~m}$ on the horizontal axis at a depth of $225 \mathrm{~m}$.

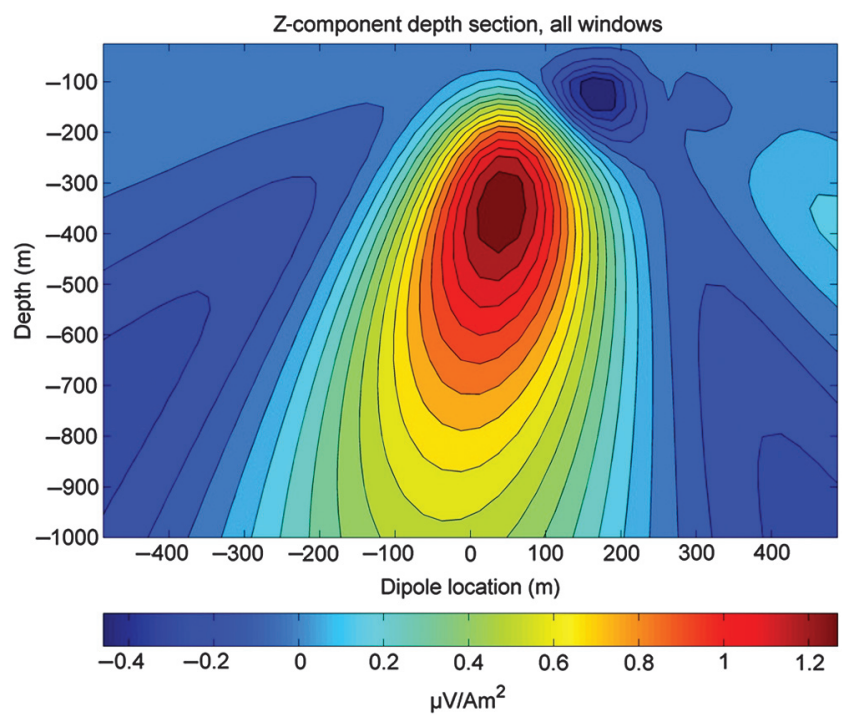

Figure 13. Horizontal dipole equivalent-dipole depth section applied to the field data. The hotter colors indicate the approximate location and apparent depth of the conductor.
The above discussion was for a horizontal dipole or vertical body. The same data could be combined using a vertical dipole field, which would couple strongly to a horizontal body. In this case, the weights applied to sum the receiver responses to show the horizontal body would be a vertical dipole field.

The method was tested on a synthetic data set for a subsurface horizontal dipole target located at position $0 \mathrm{~m}$ and a depth of $225 \mathrm{~m}$ (Figure 12). The equivalent-dipole depth section is symmetric around the lateral position of the body $(0 \mathrm{~m})$ with the maximum contour occurring between 275- and 425-m depth. Experiments with other dipole depths indicate that the depth section consistently places the top of the maximum contour interval 50-m deeper compared with the actual depth of the model, regardless of the dipoles location on the profile. This suggests that the proposed procedure images features at a greater depth that the causative body is actually located.

The field data have been processed using the above procedure; they shows an equivalent-dipole depth section with the maximum located at $+37.5 \mathrm{~m}$ along the line and a depth to the top of the maximum contour interval at $-275 \mathrm{~m}$ (Figure 13). This is in agreement with the synthetic model results and suggests that the actual body is about 225-m deep. The position of the body on the profile is consistent with the location determined from previous geologic and geophysical work. Figure 13 is for all windows (4-20) that are not affected by noise. Additional sections (not shown) were generated for windows 4-12 showing the depth of the body at $275 \pm 25 \mathrm{~m}$ for these delay times, with the lateral position at about $37.5 \mathrm{~m}$ with some delay times showing the position between 37.5 and $62.5 \mathrm{~m}$. Windows 13-16 show the depth of the body 325 to $350 \mathrm{~m}$ with the lateral position at $37.5 \pm 25 \mathrm{~m}$. Sections that only use the latest delay times (17-20) produce a section that cannot be interpreted as the signal is hidden within the noise.

The equivalent-dipole depth section generated from the field data shows a slight asymmetry with larger responses to the left. This is consistent with the geologic information, which suggests subvertical to steep dips to either the southeast or northwest (Croteau, 2009). The geologic information suggests that the body has a depth of $75 \mathrm{~m}$. However, the similarity of the equivalent-dipole depth sections with the synthetic sections suggests a greater depth. Further investigation of on-site drillhole data showed that there is some mineralization intersected at approximately 250-m depth, 175-m deeper than the target was originally thought to be. Recent work on a more sophisticated processing procedure (Kolaj and Smith, 2014) has yielded a depth estimate closer to $100 \mathrm{~m}$.

\section{CONCLUSIONS}

As shallower deposits become scarcer, there is a strong need to devise a new methodology to discover conductive ore deposits at greater depths. Using a procedure with multiple transmitter and receiver locations, the $\mathrm{S} / \mathrm{N}$ of a response can be elevated and observed to later delay times, resulting in more interpretable data. In one example, the $\mathrm{S} / \mathrm{N}$ was increased by a factor of seven by increasing the effective transmitter-loop size by a factor of four. When the effective-loop size is larger by a factor of eight using a configuration similar to the InfiniTEM configuration, the $\mathrm{S} / \mathrm{N}$ at comparable times was 144 . Using a synthetic dipole example, the response is shown to decrease as a function of increasing depth of the body. Combining receiver measurements from different transmitter positions in such a way that the $\mathrm{S} / \mathrm{N}$ increases by a factor of seven allows a vertical 
body (horizontal dipole) previously visible only to depths of $150 \mathrm{~m}$ to be seen at $300-\mathrm{m}$ depth or a larger body at $300 \mathrm{~m}$ could be seen to $575 \mathrm{~m}$. Hence, improving the $\mathrm{S} / \mathrm{N}$ means that deeper bodies can be seen.

Reciprocity can reduce the time that it takes to acquire this type of data because not all receiver positions need to be occupied if there is a reciprocal measurement. On the other hand, if reciprocal measurements are acquired, they can be used to further increase the $\mathrm{S} / \mathrm{N}$.

Equivalent-dipole depth sections can be used as a fast and easy way to display the data in such a way that the $\mathrm{S} / \mathrm{N}$ is increased and the depth and position of the body can be interpreted. However, the features imaged appear to be too deep. More sophisticated methods might be able to produce sections that more accurately reflect the depth of the body.

There is additional time and expense associated with collecting data from multiple transmitters and receivers. However, the added effort should provide the benefit of greater ability to identify deep targets, resolve the response from smaller targets, and characterize near-surface variations.

\section{ACKNOWLEDGMENTS}

We are grateful to NSERC; Vale; Sudbury Integrated Nickel Operations, a Glencore company; Wallbridge Mining; KGHM International; and the Centre for Excellence in Mining Innovation for financial support. Abitibi Geophysics was helpful through the donation of equipment and personnel, in particular, C. Malo-Lalande and D. Giroux and his crew. The authors would also like to acknowledge M. Kolaj, D. Parry, and O. Olaniyan for many helpful discussions and help with the field work.

\section{REFERENCES}

Boivin, M., 2007, Advances in geophysical technology for VMS exploration, in B. Milkereit, ed., Proceedings of Exploration 07: Fifth Decennial International Conference on Mineral Exploration, Decennial Mineral Exploration Conferences, 731-739.

Chen, J., D. W. Oldenburg, and E. Haber, 2005, Reciprocity in electromagnetics: Application to modeling marine magnetometric resistivity data: Physics of the Earth and Planetary Interiors, 150, 45-61, doi: 10.1016/ j.pepi.2004.08.015

Commer, M., S. L. Helwig, A. Hördt, C. Scholl, and B. Tezkan, 2006, New results on the resistivity structure of Merapi Volcano (Indonesia), derived from three-dimensional restricted inversion of long-offset transient electromagnetic data: Geophysical Journal International, 167, 1172-1187, doi: 10.1111/j.1365-246X.2006.03182.x.

Croteau, M., 2009, 2009 report on the Parkin offset properties Sudbury: Wallbridge Mining Company Limited.

Eaton, P., B. Anderson, S. Queen, I. Mackenzie, and D. Wynn, 2010, NEWDAS - The Newmont distributed IP data acquisition system: 80th Annual International Meeting, SEG, Expanded Abstracts, 1768-1772.

Eaton, P. A., and G. W. Hohmann, 1987, An evaluation of electromagnetic methods in the presence of geologic noise: Geophysics, 52, 1106-1126, doi: $10.1190 / 1.1442376$.
Frischknecht, F. C., V. Labson, B. R. Spies, and W. L. Anderson, 1991, Profiling methods using small sources, in M. Nabighian, ed., Electromagnetic methods in applied geophysics: SEG, 2, 105-270.

Garner, S., and D. Webb, 2000, Broadband MT and IP electrical property mapping with MIMDAS: 70th Annual International Meeting, SEG, Expanded Abstracts, 1085-1088.

Golden, H., A. Duncan, and T. Herbert, 2006, Geoferret: Rapid, low-noise deep-penetrating geophysics: Presented at the 76th Annual General Meeting, Workshop on Uranium Exploration, SEG.

Goldie, M., 2007, A comparison between conventional and distributed acquisition induced polarization surveys for gold exploration in Nevada: The Leading Edge, 26, 180-183, doi: 10.1190/1.2542448.

Halverson, M. O., J. E. E. Kingman, and J. D. Corbett, 1989, Advances in IP technology: Telluric cancellation and high spatial resolution arrays, in G. D. Garland, ed., Proceedings of Exploration '87, Special volume 3, Ontario Geological Survey, 183-190.

Harrington, R. F., 1961, Time-harmonic electromagnetic fields: McGrawHill.

Kingman, J. E. E., J. G. Donohue, and T. J. Ritchie, 2007, Distributed acquisition in electrical geophysical systems, in B. Milkereit, ed., Proceedings of Exploration 07: Fifth Decennial International Conference on Mineral Exploration, Decennial Mineral Exploration Conferences, 425-432.

Koch, R. R., and F. Dalidowicz, 1996, Shea Creek - A deep geophysical exploration discovery, in K. E. Ashton, and C. T. Harper, eds., Proceedings of MinExpo '96 symposium - Advances in Saskatchewan Geology and Mineral Exploration: Saskatchewan Geological Society, 96-108

Kolaj, M., and R. Smith, 2014, A multiple transmitter and receiver electromagnetic system for improved target detection: 84th Annual International Meeting, SEG, Expanded Abstracts, 1754-1758, doi: 10.1190/segam2014-0133 .1.

Malo Lalande, C., 2007, An InfiniTEM® survey leads to a new discovery on the Coulon Property, in B. Milkereit, ed., Proceedings of Exploration 07: Fifth Decennial International Conference on Mineral Exploration, Decennial Mineral Exploration Conferences, 1107-1110.

McMonnies, B., and V. Gerrie, 2007, Ground and borehole geophysics A decade of improvements, in B. Milkereit, ed., Proceedings of Exploration 07: Fifth Decennial International Conference on Mineral Exploration, Decennial Mineral Exploration Conferences, 39-49.

Nabighian, M., and J. Macnae, 1991, Time domain electromagnetic prospecting methods, in M. Nabighian, ed., Electromagnetic methods in applied geophysics: SEG, 2, 427-520.

Nimeck, G., and R. Koch, 2008, A progressive geophysical exploration strategy at the Shea Creek uranium deposit: The Leading Edge, 27, 52-63, doi: $10.1190 / 1.2831680$.

Parasnis, D. S., 1988, Reciprocity theorems in geoelectric and geoelectromagnetic work: Geoexploration, 25, 177-198, doi: 10.1016/0016-7142 (88)90014-2.

Polzer, B., 2000, The role of borehole EM in the discovery and definition of the Kelly Lake Ni-Cu deposit, Sudbury, Canada: 70th Annual International Meeting, SEG, Expanded Abstracts, 1063-1066.

Polzer, B., J. Macnae, Y. Lamontagne, and R. Koch, 1989, Lateral resolution enhancement of TEM data by correlation processing: 59th Annual International Meeting, SEG, Expanded Abstracts, 182-184.

Powell, B., G. Wood, and L. Bzdel, 2007, Advances in geophysical exploration for uranium deposits in the Athabasca Basin, in B. Milkereit, ed., Proceedings of Exploration 07: Fifth Decennial International Conference on Mineral Exploration, Decennial Mineral Exploration Conferences, 771-790.

Rost, S., and C. Thomas, 2002, Array seismology: Methods and applications: Reviews of Geophysics, 40, 1008, doi: 10.1029/2000RG000100.

Sheriff, R. E., 2002, Encyclopedic dictionary of applied geophysics: SEG.

Spies, B. R., 1975, The dual loop configuration of the transient electromagnetic method: Geophysics, 40, 1051-1057, doi: 10.1190/1.1440581.

Spies, B. R., 1989, Depth of investigation in electromagnetic sounding methods: Geophysics, 54, 872-888, doi: 10.1190/1.1442716.

Tirschmann, P. A., 1990, Geology report: Northbridge (6-245), Parkin/ Norman Townships.

Zhdanov, M. S., 2010, Electromagnetic geophysics: Notes from the past and the road ahead: Geophysics, 75, no. 5, 75A49-75A66, doi: 10.1190/1 .3483901 . 